\section{PROTEIN SYNTHESIS}

Chain Termination

from our Cell Biology Correspondent ANY lingering doubts about the universality of the genetic code have now been finally dispelled by Beaudet and Caskey (Proc. US Nat. Acad. Sci., 68, 619 ; 1971). They have now shown that the three codons UAA, UAG and UGA which serve as full stops, terminating the synthesis of proteins in bacteria, fulfil the same function in eukaryotic cells. Fifty-nine of the codons in eukaryotic messenger RNAs have now been assigned functions and in every case these assignments are identical to those for bacteria. In other words, bacteria and eukaryotes use identical genetic dictionaries.

Beaudet and Caskey have exploited the elegantly simple formylmethionine release assay, developed by Nirenberg's group, to prove that U.AA, UAG and UGA act as termination signals in eukaryotes. In essence. the assay involves making a complex of a ribosome, from either a bacterial or eukaryotic cell, an initiator codon AUG, which serves as messenger, and a molecule of charged bacterial initiator transfer RNA, fmet-tRNA . This complex acts as substrate and release factor(s) and the codon or tetranucleotide to be tested are added. If the tri or tetranucleotide contains a terminator

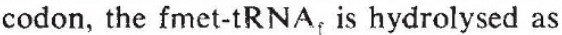
at termination and free formylmethionine is released and assayed.

Using ribosomes from rabbit reticulocytes and release factor from these cells, or from guinea-pig or Chinese hamster liver, Beaudet and Caskey found that the three tetranucleotides UAAA, UAGA and UGAA all stimulate the release of formylmethionine, whereas the tetranucleotides AAAA and UUUU containing lysine and phenylalanine codons do not. Furthermore, the trinucleotides UAA, UAG and UGA also fail to stimulate release of formylmethionine when rabbit ribosomes and mammalian release factor are used. By contrast when Escherichia coli ribosomes and release factors are used in this assay, either the tetranucleotides or the trinucleotide terminator codons stimulate release. This difference probably reflects the failure of the trinucleotides to bind to rabbit ribosomes.

The release factors in the bacterial and mammalian systems also differ. The bacterial factors have been resolved into three discrete proteins called $\mathbf{R}_{1}, \mathbf{R}_{2}$ and $\mathbf{S} . \mathbf{R}_{1}$ apparently recognizes the two terminator codons UAA and UAG and $R_{3}$ recognizes UAA and UGA. Because both factors recognize UAA, and for other reasons, it seems likely that this codon (ochre) is probably the chief terminator whereas the other two, UAG (amber) and UGA, provide accessory fail-safe signals to ensure that termination never fails. Factor $S$ comes into the picture by stimulating the rate of release catalysed by either $\mathbf{R}_{1}$ or $\mathbf{R}_{2}$. By contrast Beaudet and Caskey have failed so far to resolve mammalian release factor into components, and they have failed to find a stimulatory factor analogous to $\mathrm{S}$. The mammalian factor stimulates release with, and must therefore recognize, all three terminator codons and with a molecular weight of about 255,000 it is a much larger protein, or complex of proteins, than the bacterial $\mathrm{R}$ and $\mathrm{S}$ factors, all of which weigh about 40-50,000 daltons. Whether it will ultimately prove possible to split the mammalian release factor into functional subunits remains to be seen.

Beaudet and Caskey also report that the addition of GTP to their assay stimulates the release of formylmethionine and the GTP is hydrolysed, whereas the GTP analogue GDPCP inhibits release. A mixture of ribosomes and release factor hydrolyses GTP and the tetranucleotide UAAA stimulates this reaction and also the binding of release factor to ribosomes. Certain antibiotics block the release of formylmethionine in the release assay but do not inhibit GTP hydrolysis. It might be speculated therefore that GTP plays a part in chain termination by promoting the binding of release factor to the ribosome (messenger) nascent polypeptide complex; the release factor might then act as a trigger and cause the peptidyl transferase enzyme of the ribosome to hydrolyse the peptidyl transfer RNA, instead of catalysing the formation of a further peptide bond.

Clearly when it comes to determining the precise interaction between ribo- some, messenger and release factor it will be important to have pure release factor available, and while Beaudet and Caskey have been probing termination with mammalian release factor Klein and Cappechi (J. Biol. Chem., 246, 1055; 1971) have been purifying the bacterial $R_{1}$ and $R_{2}$ factors from the MRE 600 strain of $E$. coli. Each factor is a single polypeptide chain, $\mathbf{R}_{1}$ has a molecular weight of 44,000 and $R_{2} 47,000$, and if each has a spherical configuration the molecules must be about $47 \AA$ in diameter. From their purification data, Klein and Cappechi estimate that there are $500 R_{1}$ and $700 R_{2}$ molecules per cell compared with about 30,000 ribosomes and an even larger number of elongation factor molecules per cell.

\section{ENZYMES \\ Orbital Obit}

from our Molecular Biology Correspondent ONCE in a while one is treated to the awesome spectacle at the launching of a new concept, when, with bystanders cheering and band playing, it runs down the slipway and sinks without trace. Such, it now seems, was the fate of a theory of enzymatic catalysis propounded last year by Storm and Koshland. The hypothesis, to which they gave the euphonious name of "orbital steering", rested on measurements of reaction rates for $\gamma$-lactone formation in bicyclic compounds in which the position of the hydroxyl group was immobilized by the rigidity of the ring system. With the orbitals so aligned as to optimize the angle of attack, Storm and Koshland stated, rate increases of up to four orders of mag-

\title{
Mixmaster Universe Straightened Out
}

Cosmologists delight in constructing model universes, even if these models have little relation to the universe in which we live. One of the mathematical possibilities produced recently was a model in which particle horizons were removed, and information could be transmitted from one observer to any other observer before any given time. C. W. Misner, who put forward this model, christened it the "mixmaster universe", because this violation of the usual limits on causal interactions enables information to be spread throughout the whole universe and could (perhaps) have some bearing on the observed uniformity of the " $3 \mathrm{~K}$ " background radiation, which has the same effective temperature irrespective of which part of the sky it is coming from.

Unfortunately for this idea, it now seems that the mathematical peculiarity on which the model is founded has no valid application to our universe. M. A. H. MacCallum, of the University of Cambridge, presents in the next Nature Physical Science a discussion of the mixmaster universe problem which shows that the set of models which have no particle horizons is empty. It does still seem possible to remove the particle horizon in one direction only, at every time, but this set of models corresponds exactly with the set of rotationally symmetric models, which are already well known to cosmologists, and which even the simplest observations show to be invalid descriptions of our universe. MacCallum points out the prospect of obtaining more mathematical pleasure from this mixmaster universe problem by introducing quantum effects, a further intricate development which will have little bearing on practical astronomy. 